\title{
A NOTE ON L. E. REIZIN'S PAPER "BEHAVIOR OF INTEGRAL CURVES OF SYSTEMS OF THREE DIFFERENTIAL EQUATIONS NEAR A SINGULAR POINT"
}

\author{
R. E. ZINDLER
}

1. Introduction. This note concerns Theorem 2 in L. E. Reizin's paper [1]. It is proven in $\$ 2$ of this note that Reizin's proof of Theorem 2 is incorrect because he inferred from the statement of the theorem that a particular function $R(\phi, \psi)$ is identically one. Further, it is shown that more general $R$ 's are permissible by the conditions of the theorem and the theorem is properly restated. In $\$ 3$ the existence of a function $B(r)$, needed in the proof of Theorem 2 , is shown, in general, for conditions on two functions which are slightly more restrictive than Reizin assumes. The statement of the first part of Theorem 2 (which we call Theorem $2 \mathrm{~A}$ ) is:

TheOREM 2A. Given a system of differential equations (1) for which $\Phi \equiv \Psi \equiv 0$. If, in a certain sufficiently small neighborhood of the origin $|r| \leqq r_{0}$, it is possible to find a continuous non-negative function $B(r)$, for which

$$
\int_{0}^{r_{0}} B(r) r^{-2} d r<+\infty
$$

and

$$
|f(r, \phi, \psi)| \leqq B(r) \text { and }|g(r, \phi, \psi)| \leqq B(r),
$$

then all integral curves of the system (1) in the sufficiently small neighborhood of the origin $|r| \leqq r_{1}$ pass through the singular point with a definite tangent and there exists along each direction at least one integral curve.

The statement of the second part of Theorem 2 (which we call Theorem 2B) is:

THEOREM 2B. Given the conditions of Theorem 2A and the additional relationships

$$
\left|\frac{g(r, \phi, \psi)}{\sin \phi}\right| \leqq B(r),
$$

Presented to the Society, April 23, 1955; received by the editors April 22, 1955. 


$$
\begin{aligned}
&\left|\rho\left(r, \phi_{1}, \psi_{1}\right)-\rho\left(r, \phi_{2}, \psi_{2}\right)\right| \leqq C_{\rho} B(r)\left(\left|\phi_{1}-\phi_{2}\right|+\left|\psi_{1}-\psi_{2}\right|\right),\left|f\left(r, \phi_{1}, \psi_{1}\right)-f\left(r, \phi_{2}, \psi_{2}\right)\right| \leqq C_{f} B(r)\left(\left|\phi_{1}-\phi_{2}\right|+\left|\psi_{1}-\psi_{2}\right|\right), \\
&\left|\frac{g\left(r, \phi_{1}, \psi_{1}\right)}{\sin \phi_{1}}-\frac{g\left(r, \phi_{2}, \psi_{2}\right)}{\sin \phi_{2}}\right| \leqq C_{\diamond} B(r)\left(\left|\phi_{1}-\phi_{2}\right|+\left|\psi_{1}-\psi_{2}\right|\right),
\end{aligned}
$$

where $C_{\rho}, C_{f}, C_{o}$ are certain positive constants, then along each direction there exists only one integral curve.

The system of equations (1) is

$$
\begin{aligned}
& \frac{d x}{d t}=X(x, y, z)+\xi(x, y, z), \\
& \frac{d y}{d t}=Y(x, y, z)+\eta(x, y, z), \\
& \frac{d z}{d t}=Z(x, y, z)+\zeta(x, y, z),
\end{aligned}
$$

where $X, Y, Z$ are homogeneous polynomials of $m$ th degree relative to $x, y, z$ with constant coefficients having only one common zero at the origin and the functions $\xi, \eta, \zeta$ are continuous, satisfy

$$
\lim _{r \rightarrow 0} \frac{\xi(x, y, z)}{r^{m}}=\lim _{r \rightarrow 0} \frac{\eta(x, y, z)}{r^{m}}=\lim _{r \rightarrow 0} \frac{\zeta(x, y, z)}{r^{m}}=0
$$

where $r=\left(x^{2}+y^{2}+z^{2}\right)^{1 / 2}$ and are such functions that the origin is an isolated singular point for $r \leqq r_{0}$. A substitution

$$
x=r \sin \phi \cos \psi, \quad y=r \sin \phi \sin \psi, z=r \cos \phi
$$

is made and the system (1) becomes

$$
\begin{aligned}
\frac{d r}{d t} & =r^{m} R(\phi, \psi)+\rho(r, \phi, \psi), \\
r \frac{d \phi}{d t} & =r^{m} \Phi(\phi, \psi)+f(r, \phi, \psi), \\
r \sin \phi \frac{d \psi}{d t} & =r^{m} \Psi(\phi, \psi)+g(r, \phi, \psi),
\end{aligned}
$$

where $R, \Phi, \Psi$ are expressed by the formulae

$$
\begin{aligned}
& R(\phi, \psi)=\sin \phi \cos \psi \bar{X}+\sin \phi \sin \psi \bar{Y}+\cos \phi \bar{Z}, \\
& \Phi(\phi, \psi)=\cos \phi \cos \psi \bar{X}+\cos \phi \sin \psi \bar{Y}-\sin \phi \bar{Z}, \\
& \Psi(\phi, \psi)=-\sin \psi \bar{X}+\cos \psi \bar{Y},
\end{aligned}
$$


where $\bar{X}, \bar{Y}, \bar{Z}$ are $X, Y, Z$ evaluated at $r=1$ and where $\rho, f, g$ are expressed in terms of $\xi, \eta, \zeta$ by formulae similar to (2) only evaluated at $r=r$.

2. The proof of Theorem 2A. L. E. Reizin begins the proof of Theorem $2 \mathrm{~A}$ in the following manner: "According to the given conditions of the theorem we have

$$
X=x, \quad Y=y, \quad Z=z, \quad m=1, \quad R \equiv 1, \quad \Phi \equiv \Psi \equiv 0 ” .
$$

The conditions $\Phi \equiv \Psi \equiv 0$ are directly stated in the theorem. If one substitutes $\Phi \equiv \Psi \equiv 0$ into equations (2) and solves for $\bar{X}, \bar{Y}, \bar{Z}$ the result is

$\bar{X}=R(\phi, \psi) \sin \phi \cos \psi, \quad \bar{Y}=R(\phi, \psi) \sin \phi \sin \psi, \quad \bar{Z}=R(\phi, \psi) \cos \phi$.

There is no necessity for $R$ to be identically one from the above equations and, in fact, given any even homogeneous polynomial $P(x, y, z)$ of degree $m-1$ in $x, y, z$ which has no zeros other than the origin, it follows that a satisfactory $R$ will be

$$
R(\phi, \psi)=\frac{P(r \sin \phi \cos \psi, r \sin \phi \sin \psi, r \cos \phi)}{r^{m-1}} .
$$

It can be verified that the only admissible $R$ 's are of this form. Therefore, it follows that

$X=x P(x, y, z), \quad Y=y P(x, y, z), Z=z P(x, y, z), m$ odd and $\Phi \equiv \Psi \equiv 0$

is the more general conclusion to be drawn from the statement of Theorem 2. Reizin's proof of Theorem 2 must then be incorrect.

Since the integral condition of the function $B(r)$ in the theorem was chosen with an eye to the conclusion $m=1$, it must be modified as follows

$$
\int_{0}^{r_{0}} B(r) r^{-m-1} d r<+\infty \text {. }
$$

With this condition and the fact that $R(\phi, \psi)$ is bounded and does not vanish anywhere (thus for bounding purposes $R$ behaves like the function 1), Theorem 2 can be modified and the proof can be made in a manner analogous to that of Reizin's. The degree of analogy is so great that we shall not prove the modified theorem but rather merely state it.

THEOREM 2A (CORRECTED). Given a set of differential equations (1) for which $\Phi \equiv \Psi \equiv 0$ and if, in a certain sufficiently small neighborhood of the origin $r \leqq r_{0}$, it is possible to find a continuous non-negative function $B(r)$, for which 


$$
\int_{0}^{r_{0}} B(r) r^{-m-1} d r<+\infty
$$

and

$$
|f(r, \phi, \psi)| \leqq B(r) \text { and }|g(r, \phi, \psi)| \leqq B(r),
$$

then all integral curves of the system (1) in a sufficiently small neighborhood of the origin $r \leqq r_{1}$ pass through the singular point with a definite tangent and there exists along each direction at least one integral curve.

3. The existence of the function $B(r)$. It was suggested to the author by Dr. J. L. Brown, Jr., of the Ordnance Research Laboratory, that by slightly modifying the conditions on the functions $f(r, \phi, \psi)$ and $g(r, \phi, \psi)$ one could prove the existence of a satisfactory function $B(r)$. The added condition is Hölder continuity of the functions $f$ and $g$ as contrasted with ordinary continuity imposed on $f$ and $g$ by Reizin. We show the existence of $B(r)$ under various conditions in Lemmas I and II and then state modified Theorems $2 A$ and $2 B$ for $f$ and $g$ Hölder continuous.

Lemma I. Given $f(r, \phi, \psi)$ and $g(r, \phi, \psi)$ Hölder continuous in the variable $r$ in the region $S\left(0 \leqq r \leqq r_{0}<1,0 \leqq \phi \leqq \pi,-\pi<\psi \leqq \pi\right)$ and that

$$
\lim _{r \rightarrow 0}|f(r, \phi, \psi)| r^{-m}=\lim _{r \rightarrow 0}|g(r, \phi, \psi)| r^{-m}=0,
$$

then it follows that there exists a non-negative function

$$
B(r) \equiv \underset{\phi, \psi}{\operatorname{MAX}}(|f(r, \phi, \psi)|,|g(r, \phi, \psi)|) r^{-r / 2}
$$

for $a \gamma>0$ (chosen in the proof) such that

$$
\begin{gathered}
\int_{0}^{r 0} B(r) r^{-m-1} d r<+\infty, \\
|f(r, \phi, \psi)| \leqq B(r) \text { and }|g(r, \phi, \psi)| \leqq B(r),
\end{gathered}
$$

and

$$
\lim _{r \rightarrow 0} \frac{\underset{\phi, \psi}{\operatorname{MAX}}|f(r, \phi, \psi)|}{B(r)}=\lim _{r \rightarrow 0} \frac{\underset{\phi, \psi}{\operatorname{MAX}|g(r, \phi, \psi)|}}{B(r)}=0 .
$$

Proof. The Hölder continuity of the function $f(r, \phi, \psi)$ and the condition

$$
\lim _{r \rightarrow 0}|f(r, \phi, \psi)| r^{m}=0
$$

implies that 


$$
\lim _{r \rightarrow 0}|f(r, \phi, \psi)| r^{-m-\gamma_{1}} \leqq K_{1}>0
$$

for a particular $\gamma_{1}>0$. Similarly we have

$$
\lim _{r \rightarrow 0}|g(r, \phi, \psi)| r^{-m-\gamma_{2}} \leqq K_{2}>0
$$

for a particular $\gamma_{2}>0$. Define the functions $\bar{f}(r)$ and $\bar{g}(r)$ as follows:

(8) $\quad \bar{f}(r)=\underset{\phi, \psi}{\operatorname{MAX}}|f(r, \phi, \psi)|$ and $\bar{g}(r)=\underset{\phi, \psi}{\operatorname{MAX}}|g(r, \phi, \psi)|$,

where the maxima are taken over $0 \leqq \phi \leqq \pi$ and $-\pi<\psi \leqq \pi$ for each value of $r$ between 0 and $r_{0}$. Define

$$
\bar{h}(\boldsymbol{r}) \equiv \operatorname{MAX}(\bar{f}(\boldsymbol{r}), \bar{g}(\boldsymbol{r}))
$$

for each value of $r\left(0 \leqq r \leqq r_{0}\right)$. Consider the expression

$$
\lim _{r \rightarrow 0} \bar{h}(r) r^{-m-r} \leqq K>0 .
$$

From (9), (8), (6), and (7) it follows that the $\gamma$ for which (10) holds is $\gamma=\operatorname{MIN}\left(\gamma_{1}, \gamma_{2}\right)>0$. Define the function $B(r)$ as

$$
B(r) \equiv \bar{h}(r) r^{-r / 2} .
$$

First let us observe that $B(r)$ is non-negative and continuous in the region $S$. Second, we see that conditions (4) are satisfied (note $r<1$ ). Third, since $B(r) r^{-m-1}$ is continuous everywhere in $S$ except possibly at $r=0$ and since at $r=0$ the function $B(r) r^{-m-1}$ becomes infinite (or zero) with order greater than minus one $(-1+\gamma / 2$ to be exact), it follows that

$$
\int_{0}^{r_{0}} B(r) r^{-m-1} d r<+\infty
$$

Also, consider the limits

$$
\lim _{r \rightarrow 0} \frac{\bar{f}(r)}{B(r)} \text { and } \lim _{r \rightarrow 0} \frac{\bar{g}(r)}{B(r)} \text {. }
$$

These limits are both zero because, for example,

$$
\lim _{r \rightarrow 0} \frac{\bar{f}(r)}{B(r)}=\lim _{r \rightarrow 0} \frac{\bar{f}(r) r / 2}{\bar{h}(r)}=0
$$

since $\bar{f}(r) \leqq \bar{h}(r)$. Hence conditions (5) are satisfied. Finally we see that the definition (11) with $\gamma$ as specified in the proof agrees with that given in the statement of the lemma. The proof is complete. 
LEMMA II. Given the conditions of Lemma I and the added condition $|g(r, \phi, \psi) / \sin \phi|$ bounded in the region $S$, then a $B(r)$ can be constructed in the manner $B(r) \equiv \operatorname{MAX}_{\phi, \psi}(|f(r, \phi, \psi)|,|g(r, \phi, \psi) / \sin \phi|) r^{-r / 2}$ and will satisfy conditions

$$
\begin{gathered}
\int_{0}^{r_{0}} B(r) r^{-m-1} d r<+\infty \\
|f(r, \phi, \psi)| \leqq B(r) \quad \text { and } \quad\left|\frac{g(r, \phi, \psi)}{\sin \phi}\right| \leqq B(r),
\end{gathered}
$$

and

$$
\lim _{r \rightarrow 0} \frac{\underset{\phi, \psi}{\operatorname{MAX}}|f(r, \phi, \psi)|}{B(r)}=\lim _{r \rightarrow 0} \frac{\underset{\phi, \psi}{\operatorname{MAX}}|g(r, \phi, \psi) / \sin \phi|}{B(r)}=0 .
$$

The proof of this lemma differs from that of Lemma I only in that the condition

$$
\lim _{r \rightarrow 0}\left|\frac{g(r, \phi, \psi)}{\sin \phi}\right| r^{-m}=0
$$

is needed instead of corresponding condition (3b). The boundedness condition on $|g(r, \phi, \psi) \csc \phi|$ insures that it does not become infinite for $\phi=0$ or $\phi=\pi$. This, with condition (3b), implies condition (12). Also $g(r, \phi, \psi) \csc \phi$ is Hölder continuous of the same degree as $g(r, \phi, \psi)$. With these observations the proof of Lemma II becomes analogous to that of Lemma I.

These results and the previous observations about L. E. Reizin's Theorem 2 enable us to state modified Theorems 2A and 2B.

THEOREM 2A (MODIFIED). Given the set of differential equations (1) where $f(r, \phi, \psi)$ and $g(r, \phi, \psi)$ are Hölder continuous and for which $\Phi(\phi, \psi)$ and $\Psi(\phi, \psi)$ are identically zero where $r, \phi, \psi$ are in the region $S$, then it follows that all integral curves in a neighborhood of the origin $0 \leqq r \leqq r_{1}$ pass through the singular point with a definite tangent and at least one integral curve exists along each direction.

ThEOREM 2B (MODIFIED). Given the conditions of Theorem 2A (modified) and the additional conditions $|g(r, \phi, \psi) \csc \phi|$ bounded in $S$ and

$$
\begin{aligned}
& \left|\rho\left(r, \phi_{1}, \psi_{1}\right)-\rho\left(r, \phi_{2}, \psi_{2}\right)\right| \leqq C_{\rho} B(r)\left(\left|\phi_{1}-\phi_{2}\right|+\left|\psi_{1}-\psi_{2}\right|\right), \\
& \left|f\left(r, \phi_{1}, \psi_{1}\right)-f\left(r, \phi_{2}, \psi_{2}\right)\right| \leqq C_{f} B(r)\left(\left|\phi_{1}-\phi_{2}\right|+\left|\psi_{1}-\psi_{2}\right|\right) \text {, } \\
& \left|\frac{g\left(r, \phi_{1}, \psi_{1}\right)}{\sin \phi_{1}}-\frac{g\left(r, \phi_{2}, \psi_{2}\right)}{\sin \phi_{2}}\right| \leqq C_{0} B(r)\left(\left|\phi_{1}-\phi_{2}\right|+\left|\psi_{1}-\psi_{2}\right|\right) \text {, }
\end{aligned}
$$


where $C_{\rho}, C_{f}, C_{o}$ are certain positive constants, then it follows that along each direction there exists only one integral curve.

\section{REFERENCES}

1. L. E. Reizin, Behavior of integral curves of systems of three differential equations near a singular point, Latvijas PSR Zinătnu Akademijas Vēstis Nr2 (43) 1951 (in Russian), Mathematical Reviews vol. 15 (1954) p. 311.

Translations of this article are available by writing to the Ordnance Research Laboratory, University Park, Pennsylvania. The article is also translated in American Mathematical Society Translations, ser. 2, vol. 1, 1955, pp. 239-252.

Ordnance Research Laboratory, Pennsylvania State University

\section{A DEVELOPMENT OF CARDINALS IN "THE CON- SISTENCY OF THE CONTINUUM HYPOTHESIS"}

\section{H D SPRINKLE}

In The consistency of the continuum hypothesis the axiom of choice is used to construct the theory of powers within that of cardinals. The main purpose here is to develop just the theory of cardinals without such an axiom. This work could be formalized with the use of the predicate calculus, but, as is done in the book, the proofs will be presented rather informally. Numbering of the definitions and theorems is intended to follow closely that of the numbering in the book.

'8.2 Dfn $\alpha \simeq \bar{\alpha} \cdot \bar{\alpha} \in O n \cdot(\beta)(\beta<\bar{\alpha} . \supset . \sim(\beta \simeq \alpha)) \cdot O n=O n$.

The existence of $\bar{\alpha}$ follows from 7.7 and the unicity is evident. ${ }^{1}$ For $Y=\alpha$ and $Y=O n, \bar{Y}$ is a normal operation, since $X \in \bar{Y} . \equiv: X \in O n \cdot(\alpha)$ $\left(\alpha \simeq^{\prime} Y . \supset . X \in \alpha\right)$. Hence by M5 taking $B=O n$ there exists a function ' $N c$, over $O n$ such that ' $N c^{\prime} \alpha=\bar{\alpha}$.

Dfn ${ }^{\prime} N c^{\prime} \alpha=\bar{\alpha} \cdot{ }^{\prime} N c \mathfrak{F} \mathfrak{n O n}$.

'8.21

$\operatorname{Dfn}{ }^{\prime} N=\mathfrak{B}\left({ }^{\prime} N c\right)$.

'8.28

$$
\alpha \subseteq \beta . \supset . \bar{\alpha} \leqq \bar{\beta} .
$$

Proof. $\beta \simeq \bar{\beta}$ so each element of $\alpha$ is associated with an element of $\bar{\beta}$ by the similarity; i.e. $(\exists x)(\alpha \simeq x \subseteq \bar{\beta})$. But $x$ being a subset of $\bar{\beta}$ is

Received by the editors March 14, 1955 and, in revised form, April 25, 1955.

${ }^{1}$ A single bar placed over either a capital or lower case letter or expression has the same effect as a double bar would have in the book. 